João Mauricio Castaldelli-Maia, 1,2 Michelle B. Riba, ${ }^{3}$ Dusica Lecic-Tosevski, ${ }^{4}$ Prabha S. Chandra, ${ }^{5}$ Alfredo Cia, ${ }^{6}$ Peter J. Tyrer, ${ }^{7}$ Reinhard Heun, ${ }^{8}$ Christopher Paul Szabo ${ }^{9}$

\title{
The Main Gaps for Randomized-controlled Trials in Psychiatry: A Bibliometric Study
}

\author{
'Department of Neuroscience, ABC Health University Center, Santo André, SP, Brazil \\ 2Department of Psychiatry, Medical School, University of São Paulo, São Paulo, SP, Brazil \\ ${ }^{3}$ Department of Psychiatry, University of Michigan, Ann Arbor, MI, U.S. \\ 4Serbian Academy of Sciences and Arts, Belgrade, Serbia \\ ${ }^{5}$ Department of Psychiatry, National Institute of Mental Health and Neurosciences, Bangalore, India \\ ${ }^{6}$ Anxiety Disorders Center, Buenos Aires, Argentina \\ Centre for Psychiatry, Imperial College, London, UK \\ ${ }^{8}$ Department of Psychiatry, University of Bonn, Bonn, Germany \\ 'Department of Psychiatry, School of Clinical Medicine, Faculty of Health Sciences, University of the Witwatersrand, Johannesburg, \\ South Africa \\ *email: jmcmaia2dgmail.com
}

DOI: $10.2478 / g p-2020-0008$

Received: 21 November 2019; Accepted: 27 February 2020

\begin{abstract}
Background: There is evidence of a progressive increase in the number of Randomized Controlled Trials (RCTs) in the area of psychiatry. However, some areas of psychiatry receive more attention from researchers potentially to the detriment of others.

Methods: Aiming to investigate main gaps for RCTs in psychiatry, the present bibliometric study analysed the bi-annual and five-year rates of RCTs in the main database of medical studies (Pubmed) over the 1999-2018 period $(n=3,449)$. This analysis was carried out using the ICD-10 mental and behavioural chapter. ICD-10, was the edition of the manual used throughout the above period.

Results: Overall, after 16 years of considerable increase in the bi-annual absolute number of RCTs, there has been a slowdown in the last 4 years, similar to other medical areas. Affective, organic and psychotic disorders, and depression, schizophrenia and dementia were the top studied groups and disorders respectively - ahead of other groups/diagnoses. For substance use disorders, there has been a decrease of RCT in the last 5 years, in line with the fall of alcohol use disorder in the ranking of most studied disorders. Delirium and mild cognitive disorder are both ascending in this ranking. Personality disorders and mental retardation stand out as the least studied groups over the whole assessment period.

Conclusion: Novel treatments, ease of access to patient populations, and 'clinical vogue', seem to be more important in guiding the undertaking of RCTs than the actual need as indicated by prevalence and/or burden of disorders and public health impact. Regarding specific disorders, acute/transient psychosis; mixed anxiety and depression; adjustment disorder; dissociative and conversion disorders; somatization; hypochondria; and neurasthenia, would deserve future RCTs. Clinical researchers and editors of scientific journals should give special attention to the less studied areas and disorders, when considering conducting and publishing RCT studies, respectively.
\end{abstract}

\section{Keywords}

Bibliometric; ICD-10; mental; randomized-controlled trial; Pubmed; psychiatry

\section{INTRODUCTION}

Historically, psychiatry has been one of the areas of medicine receiving fewer resources for assistance and research, possibly due to a notable stigma within the medical field (Corrigan et al., 2005; DeLuca et al., 2017). Much of this disparity between mental and clinical conditions is due to the difficulty of including psychiatry into the medical model of care and research (Sedler, 2016). Unlike other medical areas, psychiatric diagnosis is complex because of the subjectivity inherent to psychological distress and the lack of biological markers (Möller-Leimkühler et al., 2016). The recognition of psychiatric disorders is still the subject of intense research (Möller-Leimkühler et al., 2016). The updated versions of the most important diagnostic manuals (Diagnostic and Statistical Manual of Mental Disorders - DSM and International Classification of Diseases - ICD) show great advances in the diagnostic area. These new versions (DSM-5 in 2013; and ICD-11 in 2018) also present important challenges such as the consideration of a dimensional and rather than a categorical diagnosis, giving more validity to diagnostic 
assessment in psychiatry (Castaldelli-Maia et al., 2015; 2016; 2018a), which will require further research in the coming decades.

Notwithstanding the aforementioned, the development in the area of categorical diagnosis allowed the creation of structured interviews (Shankman et al., 2018; Degenhardt et al., 2019). These psychometric instruments facilitate diagnoses listed in DSM and ICD, promoting a consistency of the diagnostic concepts both within and across countries given their translation and validation for multiple languages (Shankman et al., 2018; Degenhardt et al., 2019). Considering the important interface of mental disorders with both social and cultural aspects, the unification of the nosological concepts (mainly proposed by ICD) allowed for an operationalization of psychiatric diagnoses (Reed, 2018) and their treatment mainly through psychotherapy and psychopharmacology (Hockenberry et al., 2019; Kupfersmid, 2019; Thoma et al., 2015; Allison \& Moncrieff, 2014; Liu et al., 2017; Harris et al., 2005).

The possibility of evidence-based treatment for mental disorders has brought psychiatry closer to other medical specialties. Abolition of ineffective and disreputable treatment for patients such as hydrotherapy and bleeding, reduced the stigma attached to people with mental disorders (Overholser, 2002). Most people with mental disorders can now resume their functionality because of evidence-based treatments (Möller-Leimkühler et al., 2016). Inherent to the inclusion of psychiatry within medical research, making the conducting of Randomized Controlled Trials (RCTs) essential (Hasan et al., 2019). Currently, RCTs are critical in defining the most appropriate therapy for a particular disease (Hasan et al., 2019). With RCTs, two or more groups are randomized to receive two or more types of intervention in a controlled environment (Grossman \& Mackenzie, 2005). RCTs are of critical importance on the treatment guidelines conducting, where also a pharmaceutical industry has the interest to be on the top (e.g., Ia evidence level, A grade of recommendations). Also, RCTs are very important in the effect size calculation of a given treatment, because an absolute risk reduction is calculated from RCTs (e.g., number-needed-to-treat).

There is some evidence of a progressive increase in the number of RCTs in the area of psychiatry (Koskinen et al., 2008). There are also indications, however, that some areas of psychiatry receive more attention from researchers, potentially to the detriment of others (Koskinen et al., 2008). Bibliometric studies allow the measurement of the quantity and quality of scientific studies produced in the various areas of science (López-Muñoz et al., 2019). The former studies evaluate the trends and the concentration of scientific research, as well as the main gaps for conducting further studies (López-Muñoz et al., 2019). A detailed evaluation of RCT trends in the different areas of psychiatry would be crucial for the identification of less studied areas.

Aiming to investigate main gaps for RCTs in psychiatry, the present bibliometric study will analyse the rate of publication of RCTs in the main database of medical studies over the past 20 years (1999-2018). This analysis will be carried out as per the ICD-10 mental and behavioural chapter, which was the edition of the manual used throughout this period.

\section{METHODS}

\section{Source/Search}

The final search for RCTs published in scientific journals indexed in Pubmed was carried out on 21 $1^{\text {st }}$ March 2019 (with a final search date up to $31^{\text {st }}$ December 2018). PubMed is the most commonly used search engine in the medical sciences, being a free search engine that covers the MEDLINE database of references among others (Frandsen et al., 2019). Millions of searches are processed daily using this database, thus constituting an essential current global health tool for clinicians and researchers, with an enormous positive effect in public health (Manca et al., 2017). We selected Pubmed among the available databases because a previous study found that using data sources beyond PubMed has a modest impact on the results of systematic reviews of medical studies, especially when RCTs are concerned (Halladay et al., 2015). Compared to the other most popular databases in the field (Web of Science, GoogleScholar and Scopus), Pubmed presents a series of advantages such as free access, update frequency, early articles, and high standards of journal quality (Falagas et al., 2008).

\section{Inclusion/Exclusion criteria}

The fifth chapter of the tenth revision of the International Statistical Classification of Diseases and Related Health Problems (ICD-10) published by the World Health Organization (WHO) contains the Mental and Behavioural Disorders (WHO, 1992). From 1992 to 2018, it was the official mental health system for almost all countries, including the USA, (WHO, 1992; Reed, 2010). Two separate publications of this chapter were made available (WHO, 1992; 1993). The 'blue book' contained the clinical descriptions and diagnostic guidelines for clinical and general educational use (WHO, 1992). The green book contained the diagnostic criteria for research (WHO, 1993). Both books list the following eleven groups of 
disorders: [F00-F09] organic, including symptomatic, mental disorders; [F10-F19] mental and behavioural disorders due to psychoactive substance use; [F20-F29] schizophrenia, schizotypal and delusional disorders; [F30-F39] mood (affective) disorders; [F40-F48] neurotic, stress-related and somatoform disorders; [F50-F59], behavioural syndromes associated with physiological disturbances and physical factors; [F60-F69] disorders of adult personality and behaviour; [F70F79] mental retardation; [F80-F89] disorders of psychological development; [F90-F98] behavioural and emotional disorders with onset usually occurring in childhood and adolescence; [F99] unspecified mental disorder.

For the present analysis, all the diagnoses included in the first eight sections [F00-F79] were included in the bibliometric analysis. The penultimate section [F80-F98] was excluded because of including specific diagnoses for child and adolescent only (i.e., developmental disorders; autism; disturbance of activity and attention; conduct disorder), because the present study aimed to compare diagnoses that were not restricted to an age interval. The last section [F99] was excluded because of the non-utility of such diagnoses (unspecified mental disorder) for RCTs.

\section{Search terms}

We included the term that referred to the diagnosis exactly as listed on the ICD-10 (WHO, 1992) for each disorder. Whenever possible, we added such terms in a Boolean search, using AND, OR, and wildcards operators, to make search as simple as possible. In addition, we allowed some well-established acronyms of ICD-10 diagnoses (i.e., anxiety disorders). We conducted the search per group of disorders, as follows:

- Organic, including symptomatic, mental disorders [F00-F09]: dementia [F01, F02, F03]; organic AND (amnesic or hallucinosis or catatonic or delusional or schizophrenia or mood or affective or anxiety or dissociative or emotionally labile or asthenic or mental or brain syndrome or personality disorder) [F06.0, F06.1, F06.2, F06.3, F06.4, F06.5, F06.6, F07.0]; delirium [F05]; mild cognitive[F06.7]; (specified or unspecified) AND mental AND brain damage [F06.8, F06.9]; (postencephalitic OR postconcussional) AND syndrome [F07.1, F07.2]; unspecified (organic personality or behavioural disorders) [F07.8, F07.9]; unspecified (organic or symptomatic) mental disorder [F09].

- Mental and behavioural disorders due to psychoactive substance use [F10-F19]: alcohol AND (intoxication OR shortterm effects OR harmful OR abuse OR misuse OR dependenc ${ }^{\star}$ OR withdrawal OR psycho ${ }^{*}$ OR amnesic) [F10.0, F10.1, F10.2, F10.3, F10.4, F10.5, F10.6, F10.7]; (tobacco OR nicotine) AND (intoxication OR short-term effects OR harmful OR abuse OR misuse OR dependenc ${ }^{*}$ OR withdrawal OR psycho* OR amnesic) [F17.0, F17.1, F17.2, F17.3, F17.4, F17.5, F17.6, F17.7]; (opioid ${ }^{\star} \mathrm{OR}_{\text {cannab }}{ }^{\star}$ OR marijuana OR sedative ${ }^{\star} \mathrm{OR}$ hypnotic ${ }^{\star}$ OR benzodiazepine ${ }^{\star} \mathrm{OR}$ cocaine $\mathrm{OR}$ stimulant ${ }^{\star} \mathrm{OR}$ caffeine OR hallucinogen ${ }^{\star} \mathrm{OR}$ volatile solvent ${ }^{\star} \mathrm{OR}$ inhalant ${ }^{\star}$ OR multiple drug use) AND (intoxication OR short-term effects OR harmful OR abuse OR misuse OR dependenc* OR withdrawal OR psycho* OR amnesic) [Flx.0, Flx.1, Flx.2, Flx.3, Flx.4, Flx.5, Flx.6, Flx.7]; delirium tremens [F10.4]; Korsakoff [F10.6]; post hallucinogen perception disorder [F16.6]; (unspecified or other) AND (mental or behavioural) disorder [Flx.8, Flx.9].

- Schizophrenia, schizotypal and delusional disorders [F20-F29]: schizophreni ${ }^{*}$ [F20.0, F20.1, F20.2, F20.3, F20.4, F20.5, F20.6, F20.7, F20.8, F23.2]; schizotypal [F21]; delusional [F22]; involutional paranoid state [F22.8]; paranoia querulans [F22.8]; (acute OR transient OR nonorganic) AND psychotic [F23, F28, F29]; acute polymorphic psychotic disorder [F23.0, F23.1]; induced delusional disorder [F24]; folie à deux [F24]; schizoaffective [F25]; chronic hallucinatory psychosis [F28].

- Mood (affective) disorders [F30-39]: (manic OR mania OR hypomania OR bipolar disorder ${ }^{\star}$ OR BD) [F30, F31]; depressive disorder or depression [F32, F33, F38.1]; persistent (mood or affective) [F34]; cyclothymia [F34.0]; dysthymia [F34.1]; mixed affective episode [F38.0]; unspecified (mood or affective) disorder [F39].

- Neurotic, stress-related and somatoform disorders [F40-F48]: phobic [F40, F40.8, F40.9]; agoraphobia [F40.0]; phobia $^{*}$ [F40.1, F40.2, F40.9]; anthropophobia [F40.1]; acrophobia OR claustrophobia [F40.2]; panic disorder [F41.0]; anxiety disorder or GAD [F41.1]; obsessivecompulsive disorder or OCD [F42]; acute stress reaction [F43.0]; post-traumatic stress disorder or PTSD [F43.1]; adjustment disorder [F43.2]; dissociative disorder [F44, F44.0, F44.1, F44.2, F44.4, F44.5, F44.6, F44.7, F44.8, F44.9]; (trance OR possession) disorders [F44.3]; Ganser's syndrome [F44.8]; multiple personality [F44.8]; somatoform disorders [F45, F45.1, F45.4, F45.8, F45.9]; somatization disorder [F45.0]; Briquet's disorder [F45.0]; multiple psychosomatic disorder [F45.0]; hypochondria* [F45.2]; body dysmorphic disorder or dysmorphophobia or nosophobia [F45.2]; somatoform autonomic dysfunction[F45.3]; (cardiac OR gastric) neurosis [F45.2]; Da Costa’s syndrome [F45.2]; neurocirculatory asthenia [F45.2]; psychalgia [F45.4]; neurotic disorders [F48, F48.8, F48.9]; neurasthenia [F48.0]; depersonalization or derealization [F48.1]; Dhat syndrome OR occupational neurosis OR psychastheni ${ }^{\star}$ OR psychogenic syncope [F48.2]; neurosis [F48.9]. 
- Behavioural syndromes associated with physiological disturbances and physical factors [F50-F59]: (eating disorder ${ }^{\star}$ OR anorexia nervosa OR bulimia nervosa OR (vomiting AND psychological)) [F50]; sleep disorder* [F51]; insomnia [F51.0]; hypersomnia [F51.1]; sleep-wake schedule[F51.2]; sleepwalking OR somnambulism [F51.3]; sleep terrors OR night terrors[F51.4]; nightmares [F51.5]; sexual dysfunction [F52, F52.8, F52.9]; frigidity [F52.0]; hypoactive sexual desire disorder [F52.0]; anhedonia sexual [F52.1]; failure of genital response [F52.2]; female sexual arousal disorder [F52.2]; male erectile disorder [F52.2]; psychogenic impotence [F52.2]; orgasmic dysfunction [F52.3]; psychogenic anorgasmy [F52.3]; premature ejaculation [F52.4]; nonorganic AND (vaginismus OR dyspareunia) [F52.5, F52.6]; excessive sexual drive[F57.2]; (mental OR behavioural) AND puerperium [F53]; (postnatal OR postpartum) depression [F53.0]; puerperal psychosis [F53.1]; (psychological or behavioural) AND (disorders or diseases) AND elsewhere [F54, F59]; abuse AND non-dependence-producing [F55].

- Disorders of adult personality and behaviour [F60-F69]: personality disorder* $[\mathrm{F} 60, \mathrm{~F} 60.1, \mathrm{~F} 60.2, \mathrm{~F} 60.3, \mathrm{~F} 60.4$, F60.5, F60.6, F60.7, F60.8, F60.9, F61]; enduring personality changes [F62]; (habit OR impulse) disorder [F63]; pathological gambling [F63.0]; pathological fire-setting OR pyromania [F63.1]; pathological stealing OR kleptomania [F63.2]; trichotillomania [F63.3]; intermittent explosive disorder [F63.8]; gender identity disorder [F64, F64.2]; transsexualism [F64.0]; dual-role transvestism [F64.1]; disorders of sexual preference [F65, F65.6, F65.8]; fetishes ${ }^{*}$ [F65.0, F65.1]; exhibitionism [F65.2]; voyeurism [F65.3]; paedophilia [F65.4]; sadomasochism [F65.5]; frotteurism [F65.8]; necrophilia [F65.8]; zoophilia [F65.8]; psychosexual development disorders [F66, F66.8, F66.9]; sexual maturation disorder [F66.0]; ego-dystonic sexual orientation [F66.1]; sexual relationship disorder [F66.2]; elaboration of physical symptoms for psychological reasons [F68.0]; factitious disorder [F68.1]; Munchausen syndrome [F68.1]; (other specified OR unspecified) disorders adult (personality or behaviour) [F68.8, F68.9].

- Mental retardation [F70-F79]: mental retardation [F70, F71, F72, F73, F78, F79].

Pubmed filters (Hoogendam et al., 2009) were initially used to restrict the search as follows:

- Species: restricted to 'humans' only;

- Article type: restricted to 'clinical trials' only;

- Search field: title only;

- Publication date: specific desired range from January $1^{\text {st }}$, 1999 to December 31 ${ }^{\text {st }}, 2018$;
- Key-word: (randomized OR randomised) plus the search terms described in the preceding section.

\section{Data Items}

Number of studies published were registered by each search term $(n=123)$ within the 7 disorder groups listed above, in different time frames. First, the number of studies published by five-years periods (1999-2003; 2004-2008; 2009-2013; 2014-2018). Second, the number of studies published by the 7 disorder groups during each period of 2 years within the last 20 were registered (1999-2000; 2001-2002; 2003-2004; 2005-2006; 2007-2008; 2009-2010; 2011-2012; 2013-2014; 2015-2016; 2017-2018). Third, the first two steps were repeated including all the search terms, aiming to register the number of all the psychiatric disorders together. Finally, these steps were repeated without any search term, aiming to register the total number of studies published in the entire Pubmed during the same timeframes.

\section{Statistical Analysis}

Considering that we aimed to investigate the main gaps for RCTs in psychiatry, we conducted multinomial logistic regression models to compare the distribution of RCTs in each 5-year period for ICD-10 mental and behavioural group using STATA software version 11.2. We carried out three different models, using different reference categories in each one (Model 1 = F30-F39; 1999-2003; Model 2 = F20-F29; 2004-2008; Model 3 = F00-F09; 2009-2013). The same approach was used to compare mental health RCTs versus RCTs in other medical areas, always having the other medical areas as the reference category for the group, but changing period reference category (Model $4=1999-2003$; Model $5=2004-2008$; Model $6=2009$ 2013). We changed the reference groups to investigate all the differences among time periods and groups of disorder.

In addition, a logarithmic function was used to graphically plot the bi-annual rates of RCTs per mental disorder group, mental disorders as a whole, and other than mental health areas. The logarithmic function enabled the comparison of all the curves together in the same graph (reduced the overall and increased readability).

\section{RESULTS}

Table 1 presents a comparative distribution of RCTs published in Pubmed per ICD-10 mental and behavioural chapter, per each 5 -year period during the last 20 years $(n=3,449)$. Comparing the proportion of psychiatric RCTs per group, there has been a 


\begin{tabular}{|c|c|c|c|c|c|c|c|c|c|c|c|c|c|}
\hline \multirow{5}{*}{ 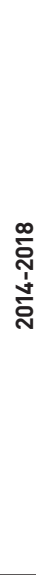 } & 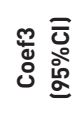 & $\stackrel{\overleftarrow{\Phi}}{\propto}$ & 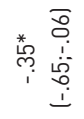 & 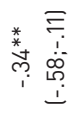 & 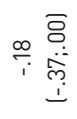 & 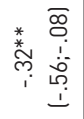 & 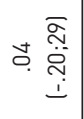 & 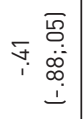 & $\dot{\Delta}$ & 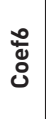 & 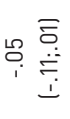 & $\stackrel{\bar{\Phi}}{\widetilde{\Phi}}$ & \\
\hline & 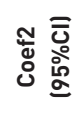 & 粒 & m. & $\underset{\widetilde{\Phi}}{\overleftarrow{\Phi}}$ & 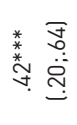 & 弥 & 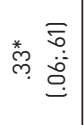 & 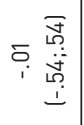 & $\dot{\Delta}$ & 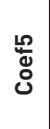 & 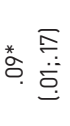 & 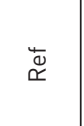 & \\
\hline & 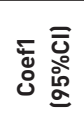 & 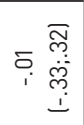 & 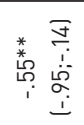 & 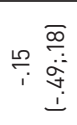 & $\stackrel{\overleftarrow{\Phi}}{\mathscr{\Phi}}$ & 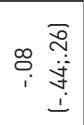 & 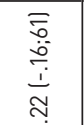 & 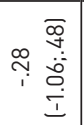 & $\stackrel{\dot{c}}{z}$ & 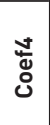 & 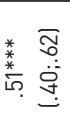 & 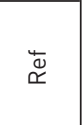 & \\
\hline & $\frac{F}{\circ}$ & $\begin{array}{l}\stackrel{\circ}{a} \\
\dot{j}\end{array}$ & i̊ & 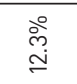 & 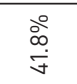 & 离 & ؛े & $\stackrel{\circ}{\stackrel{2}{2}}$ & $\begin{array}{l}\circ \\
\stackrel{\circ}{\circ} \\
\circ\end{array}$ & $\frac{\text { 풍 }}{\circ}$ & مें & 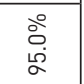 & \\
\hline & c & ले & $\stackrel{\infty}{\stackrel{\infty}{c}}$ & ఫ్ & 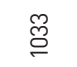 & $\stackrel{\infty}{\stackrel{\infty}{\sim}}$ & 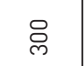 & $\bar{\sigma}$ & 0 & $=$ & $\overline{\widetilde{\infty}}$ & 岕 & \\
\hline \multirow{5}{*}{ 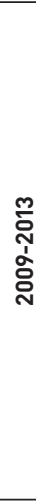 } & 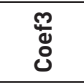 & 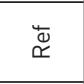 & $\stackrel{\bar{\Phi}}{\propto}$ & $\stackrel{\overleftarrow{\Phi}}{\widetilde{\Phi}}$ & $\stackrel{\bar{\Phi}}{\propto}$ & 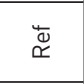 & $\stackrel{\bar{\Phi}}{\propto}$ & $\stackrel{\bar{\Phi}}{\stackrel{\Phi}{\propto}}$ & $\stackrel{\dot{s}}{z}$ & ¿ัँ & $\begin{array}{l}\bar{\Phi} \\
\widetilde{\alpha}\end{array}$ & $\stackrel{\bar{\Phi}}{\propto}$ & \\
\hline & 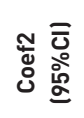 & 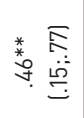 & m. & 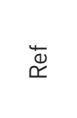 & 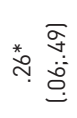 & 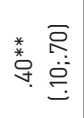 & 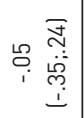 & 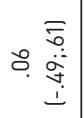 & $\dot{z}$ & 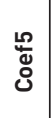 & 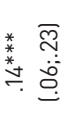 & $\stackrel{\bar{\Phi}}{\propto}$ & \\
\hline & 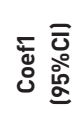 & 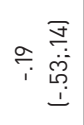 & 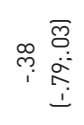 & 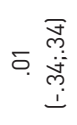 & $\underset{\widetilde{\Phi}}{\overleftarrow{\Phi}}$ & ১. & 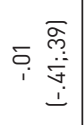 & 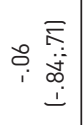 & $\dot{s}$ & 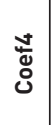 & 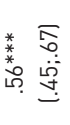 & $\underset{\propto}{\stackrel{\Phi}{\propto}}$ & \\
\hline & $\stackrel{\mp}{\circ}$ & $\stackrel{\text { ले }}{\stackrel{\circ}{ }}$ & \begin{tabular}{l}
$\circ$ \\
\hdashline
\end{tabular} & 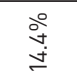 & 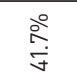 & $\begin{array}{l}\stackrel{\text { ले }}{\text { ले }}\end{array}$ & 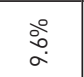 & $\stackrel{\circ}{\stackrel{\sim}{\circ}}$ & $\begin{array}{l} \\
\circ \\
\circ\end{array}$ & $\frac{\#}{\circ}$ & 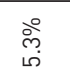 & 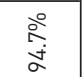 & \\
\hline & $=$ & $\stackrel{\mathscr{N}}{\underset{N}{*}}$ & $\bar{\beth}$ & 胥 & 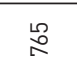 & \& & 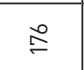 & ळ & 0 & $=$ & 尺 & 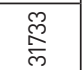 & \\
\hline \multirow{5}{*}{ 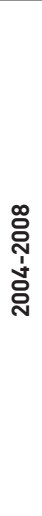 } & 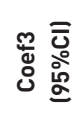 & 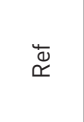 & 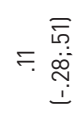 & 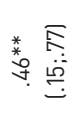 & 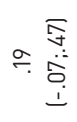 & ه. & 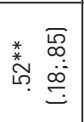 & 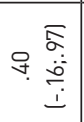 & $\stackrel{\dot{a}}{z}$ & $\begin{array}{l}\stackrel{\circ}{\circ} \\
\stackrel{0}{0}\end{array}$ & 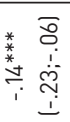 & 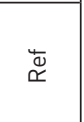 & 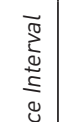 \\
\hline & 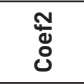 & $\stackrel{\overleftarrow{\Phi}}{\propto}$ & $\underset{\mathscr{\Phi}}{\overleftarrow{q}}$ & $\stackrel{\overleftarrow{\Phi}}{\widetilde{\Phi}}$ & 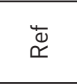 & $\stackrel{\overline{\mathbb{Q}}}{\widetilde{\alpha}}$ & 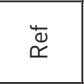 & $\stackrel{\overline{\mathbb{Q}}}{\stackrel{4}{\propto}}$ & $\dot{z}$ & 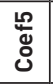 & $\stackrel{\bar{\Phi}}{\widetilde{\alpha}}$ & $\stackrel{\bar{\Phi}}{\propto}$ & 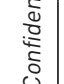 \\
\hline & 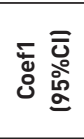 & 㒸 & 芦 & 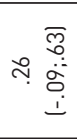 & 过 & 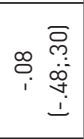 & 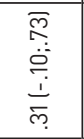 & 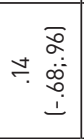 & $\stackrel{\dot{s}}{z}$ & 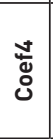 & 絭笶 & $\stackrel{\bar{\Phi}}{\propto}$ & 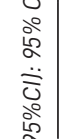 \\
\hline & $\frac{F}{\circ}$ & $\begin{array}{l}\stackrel{\circ}{\infty} \\
\text { ळ. }\end{array}$ & 今̊ & $\frac{\stackrel{\circ}{\stackrel{\circ}{\infty}}}{\stackrel{0}{\infty}}$ & $\begin{array}{l}\text { oे } \\
\text { ợ }\end{array}$ & $\stackrel{\stackrel{\circ}{\rightleftharpoons}}{=}$ & $\stackrel{\circ}{\stackrel{\circ}{ㅇ}}$ & ণัे & $\stackrel{\circ}{\circ}$ & $\frac{\overline{\#}}{\circ}$ & 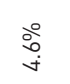 & $\begin{array}{l}\stackrel{\circ}{\circ} \\
\text { گٌ }\end{array}$ & 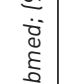 \\
\hline & c & $\bar{\sigma}$ & 员 & $\stackrel{?}{?}$ & 今 & $\stackrel{\stackrel{8}{\circ}}{\circ}$ & $\stackrel{\mathbb{N}}{ }$ & $\stackrel{\sim}{~}$ & - & $=$ & ন & $\begin{array}{l}\stackrel{\alpha}{\sigma} \\
\stackrel{\alpha}{\sigma}\end{array}$ & 㤩 \\
\hline \multirow{6}{*}{ 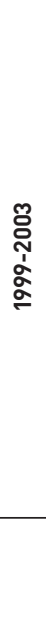 } & 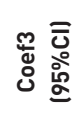 & $\underset{\mathbb{\Phi}}{\overleftarrow{\Phi}}$ & 赵 & 离 & 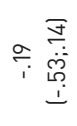 & 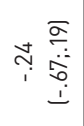 & 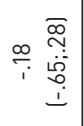 & 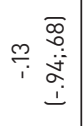 & $\stackrel{\dot{a}}{z}$ & 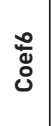 & 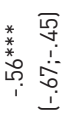 & $\stackrel{\bar{\Phi}}{\propto}$ & 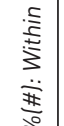 \\
\hline & 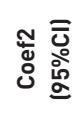 & 晜 & 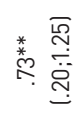 & 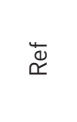 & 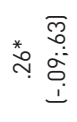 & 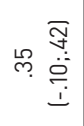 & 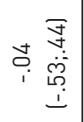 & 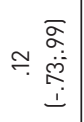 & $\stackrel{\dot{c}}{\mathrm{z}}$ & $\begin{array}{l}\frac{L}{0} \\
\stackrel{0}{0}\end{array}$ & 絭离 & $\stackrel{\overleftarrow{\Phi}}{\propto}$ & 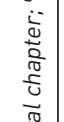 \\
\hline & ¿্ষ & $\stackrel{\overleftarrow{\Phi}}{\check{\Phi}}$ & $\underset{\widetilde{\Phi}}{\bar{\Phi}}$ & $\stackrel{\overleftarrow{\Phi}}{\propto}$ & $\underset{\widetilde{\Phi}}{\bar{\Phi}}$ & $\stackrel{\bar{\Phi}}{\simeq}$ & 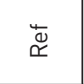 & 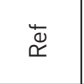 & $\stackrel{\dot{a}}{z}$ & 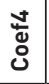 & $\underset{\widetilde{\Phi}}{\overleftarrow{\Phi}}$ & 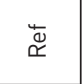 & 恿 \\
\hline & $\frac{\mp}{\circ}$ & 犬̀ & ڤે̀ & 웅 & 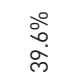 & ঐें & ڤั̀ & ஓे & $\begin{array}{l}\circ \\
\text { 今. } \\
\circ\end{array}$ & $\frac{\overline{\#}}{\circ}$ & $\stackrel{\circ}{\stackrel{一}{\circ}}$ & $\begin{array}{l}\partial^{\circ} \\
\sigma^{\circ}\end{array}$ & 竧 \\
\hline & $=$ & if & $\stackrel{\leftrightarrow}{\mathrm{m}}$ & ஸิ & Б5 & \& & $\stackrel{\leftrightarrow}{m}$ & $\infty$ & 0 & $=$ & $\stackrel{\circ}{\circ}$ & $\begin{array}{l}\stackrel{\mathscr{L}}{\infty} \\
\stackrel{\infty}{\rightleftharpoons}\end{array}$ & $\begin{array}{l}\bar{\Phi} \\
\Phi\end{array}$ \\
\hline & 옹 옹 & 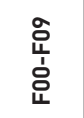 & $\frac{O}{\frac{0}{4}}$ & 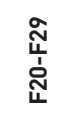 & 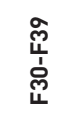 & 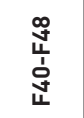 & 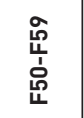 & 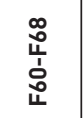 & 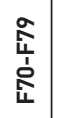 & $\begin{array}{l}0 \\
\stackrel{0}{0} \\
\stackrel{0}{0}\end{array}$ & $\begin{array}{l}\overrightarrow{\widetilde{J}} \\
\frac{\tilde{L}}{\Sigma} \\
\overline{\tilde{\omega}}\end{array}$ & 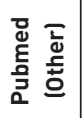 & 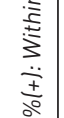 \\
\hline
\end{tabular}

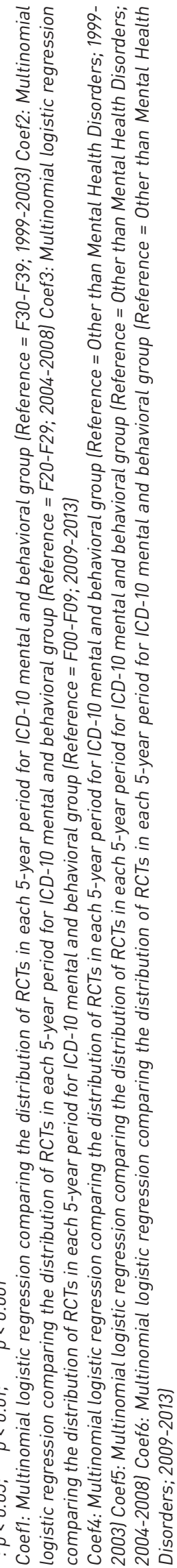




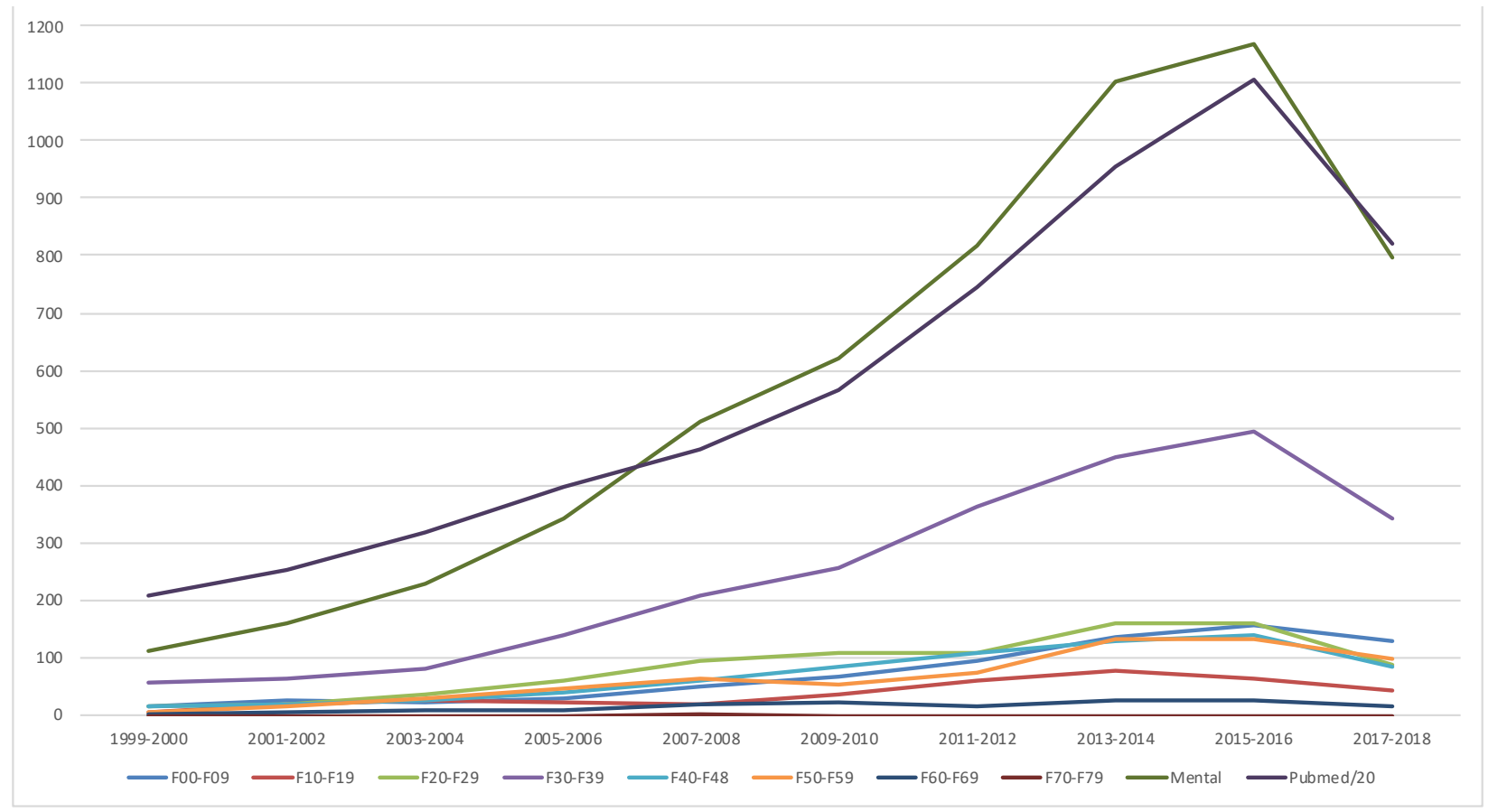

Figure 1. Frequencies of numbers of randomized-controlled trials published in Pubmed per ICD-10 mental and behavioral chapter, in the last 20 years

significant and progressive increase for organic disorders [F00F09] in the last 10 years, as compared to 2004-2008 period. The opposite situation occurred for psychotic disorders, which had significantly less studies in the last 10 years, as compared to 2004-2008. Substance use disorders [F10-F19] had a significant reduction of RCTs in 2004-2008, followed by stability in 2009-2013, and reduced again in 2014-2018 period, which was almost the opposite to behavioural syndromes associated with physiological disturbances and physical factors [F50-F59] (increase, decrease and increase). Both affective [F30-F39] and neurotic, stress-related and somatoform disorders [F40-F48] presented significant increase in 2009-2013, followed by a decrease in 2014-2018 in the number of RCTs. No significant change was found for personality disorders during the entire period.

Figure 1 presents the crude distribution of RCTs published in Pubmed per ICD-10 mental and behavioural disorder group, over the last 20 years. Overall, after 16 years of considerable increase in the bi-annual absolute number of RCTs, there has been a slowdown in the last 4 years. This trend was in line with the rest of RCTs published in Pubmed within the same period. Affective disorders [F30-F39] were the mental disorder group with more RCTs in psychiatry during these last two decades. In the beginning of the period, there were around 100 Psychiatric RCTs (around 1/40 of all the RCTs in Pubmed). Psychiatry peaked at almost 1,200 RCTs in 2015-2016 (more than 10 times higher than in 1999-2000). From 2007-2008 to 2015-2016, psychiatric RCTs were responsible for more than $1 / 20$ of all the RCTs in Pubmed. Affective disorders were responsible for $40-50 \%$ of the psychiatric RCTs during this period, peaking at almost 500 RCTs in 2015-2016 (more than 10 times higher than in 1999-2000).

Figure 2 presents the distribution of RCTs published in Pubmed per ICD-10 mental and behavioural group, over the last 20 years, in a logarithmic scale. Organic [F00-F09], psychotic [F20F29], neurotic/anxiety [F40-F48], and physiological-related [F50-F59] disorders had approximately similar trends during the last 2 decades. Psychotic disorders [F20-F29] were the second most studied psychiatric group through RCTs during almost the entire period. In the last two years, organic disorders [F00-F09] became the second most common psychiatric group with RCTs, seemingly associated with a notable decrease of the psychotic disorder group. Initially (1999-2004), the same trend of organic [F00-F09], psychotic [F20-F29], neurotic/anxiety [F40-F48], and physiological-related [F50-F59] disorders, substance use disorders [F10-F19] recorded a decrease during 2005-2008, followed by an increase (2009-2014) and another decrease in the last 4 years. Personality disorders [F60-F69] and mental retardation [F70-F79] comprise the least studied groups through RCTs during the entire period. However, the 


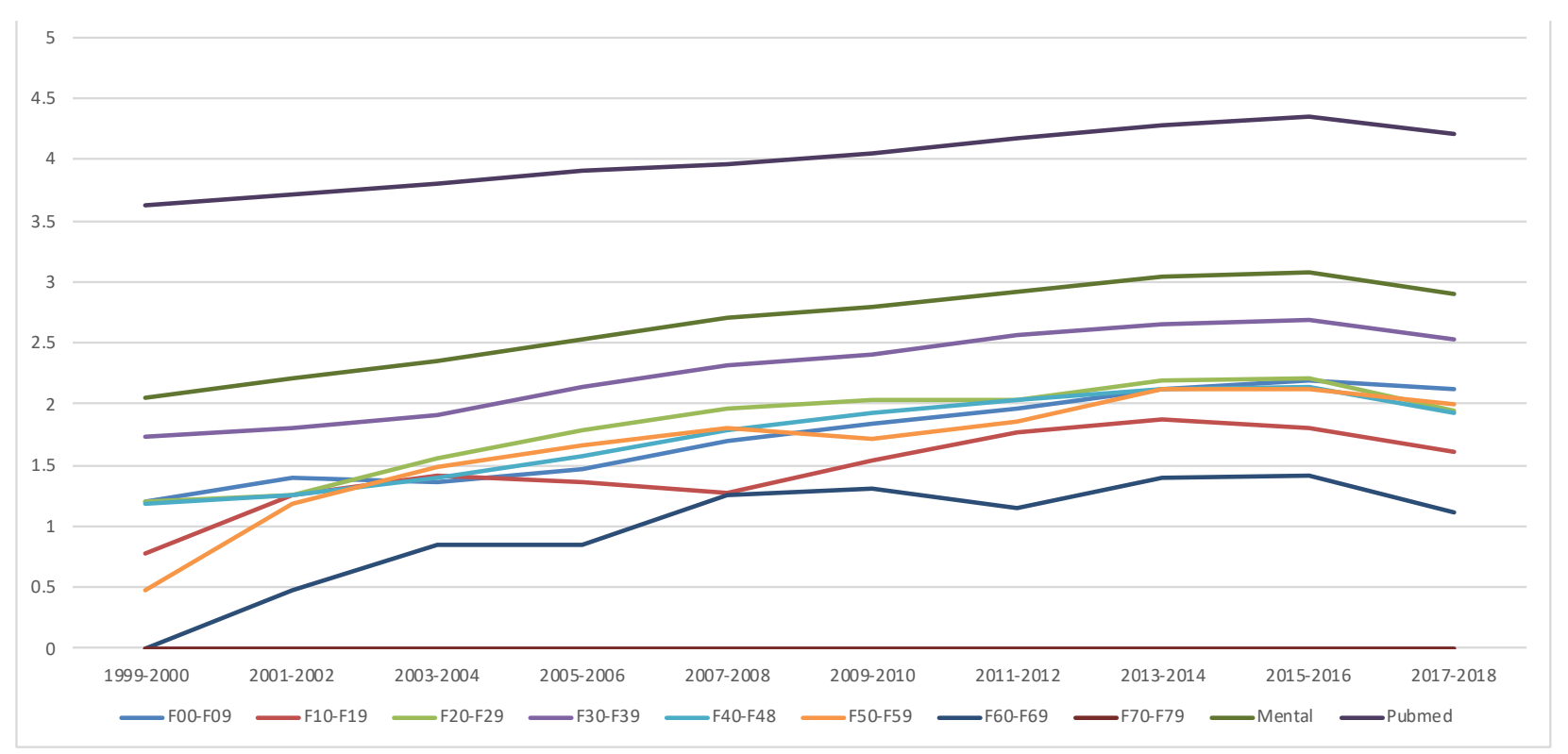

Figure 2. Frequencies of numbers of randomized-controlled trials published in Pubmed per ICD-10 mental and behavioral chapter, in the last 20 years, in a logarithmic scale ly axis indicates the power to the basis 10)

personality disorder group [F60-F69] showed a small, but linear, increase until 2007-2008, from when there has been stability of about 20 RCTs each two years. Mental retardation [F70-F79] has virtually been not studied through RCTs during the entire period.

Figure 3 presents distribution of RCTs published in Pubmed per top 20 ICD-10 mental and behavioural disorders, in the last $20,10,5$, and 2 years. In all the timeframes, the top 4 disorders did not change: depression, schizophrenia, dementia, and insomnia. The difference between depression and schizophrenia has increased, with the first being investigated more than three times that of the second in the last 2 years. Despite having exchanged places, eating, generalized anxiety and post-traumatic stress disorders remained from $6^{\text {th }}$ to $10^{\text {th }}$ places in all the timeframes. There was the same situation for drug use, obsessive-compulsive, personality, and postpartum depression disorders, which remained from $11^{\text {th }}$ to $15^{\text {th }}$ places; and for tobacco use, phobias, and sexual dysfunction, which remained from $16^{\text {th }}$ to $20^{\text {th }}$. The most remarkable changes were seen for delirium, mild cognitive, bipolar, and alcohol use disorders. Delirium and mild cognitive disorders have been increasingly more investigated through RCTs, being in the $11^{\text {th }} / 14^{\text {th }}, 10^{\text {th }} / 13^{\text {th }}, 8^{\text {th }} / 13^{\text {th }}$, and $5^{\text {th }} / 10^{\text {th }}$ places in the last 20,10 , 5 , and 2 years respectively. Whereas bipolar and alcohol use disorder have been decreasingly and less investigated through RCTs, being in the $5^{\text {th }} / 8^{\text {th }}, 6^{\text {th }} / 9^{\text {th }}, 7^{\text {th }} / 10^{\text {th }}$, and $8^{\text {th }} / 13^{\text {th }}$ places in the last 20, 10, 5, and 2 years respectively. Panic and schizoaffective disorders were not included in the top 20 in the last 2 years, although sleep and premature ejaculation disorders entered the top 20 in the same period.

\section{DISCUSSION}

The main objective of the present study was to identify the distribution and trends of psychiatric RCTs published during the last 20 years. This appears to be the first bibliometric study in the psychiatric field to explore all the ICD-10 mental and behavioural adult disorders. Overall, after 16 years of considerable increase in the bi-annual absolute number of RCTs, there has been a slowdown in the last 4 years. Affective, organic and psychotic disorders, and depression, schizophrenia and dementia were the top studied groups and disorders respectively - ahead of other groups/diagnoses. For substance use disorders, there has been a decrease in the last 5 years, in line with the fall of alcohol use disorder in the ranking of most studied disorders. Delirium and mild cognitive disorder are both ascending in this ranking, in line with a significant progressive increase of organic disorders in the proportion of RCTs per group of the ICD-10 fifth chapter. Personality disorders and mental retardation have consistently been and remain the least studied groups.

The current study demonstrated that the slowdown of RCTs in psychiatry follows a similar trend for variation of RCTs in the entire medical field, reducing increase rate in 2015-2016, and 


\begin{tabular}{|c|c|c|c|c|c|c|c|}
\hline \multicolumn{2}{|c|}{ Last 20 years } & \multicolumn{2}{|c|}{ Last 10 years } & \multicolumn{2}{|c|}{ Last 5 years } & \multicolumn{2}{|c|}{ Last 2 years } \\
\hline Disorder & $n$ of RCT & Disorder & $\mathrm{n}$ of RCTs & Disorder & $\mathrm{n}$ of RCTs & Disorder & $\mathrm{n}$ of RCTs \\
\hline Depression & 1989 & Depression & 1544 & Depression & 898 & Depression & 307 \\
\hline Schizophrenia & 761 & Schizophrenia & 551 & Schizophrenia & 297 & Schizophrenia & 88 \\
\hline Dementia & 457 & Dementia & 345 & Dementia & 207 & Dementia & 68 \\
\hline Insomnia & 256 & Insomnia & 201 & Insomnia & 131 & Insomnia & 44 \\
\hline Bipolar Disorders & 243 & $G A D$ & 186 & Eating Disorders & 109 & Delirium & 41 \\
\hline Eating Disorders & 233 & Bipolar Disorder & 173 & $-G A D$ & 100 & Eating Disorders & 35 \\
\hline$G A D$ & 225 & Eating Disorders & 169 & -Bipolar Disorders & 88 & $G A D$ & 32 \\
\hline Alcohol Use Disorders & 174 & PTSD & 135 & Delirium & 79 & -Bipolar Disorders & 30 \\
\hline PTSD & 159 & Alcohol Use Disorders & 129 & PTSD & 78 & PTSD & 25 \\
\hline Drug Use Disorders & 143 & Delirium & 107 & Alcohol Use Disorders & 61 & Mild Cognitive Disorder & 23 \\
\hline Delirium & 120 & Drug Use Disorders & 102 & Drug Use Disorders & 59 & Drug Use Disorders & 19 \\
\hline$O C D$ & 120 & $O C D$ & 94 & $O C D$ & 58 & $O C D$ & 19 \\
\hline Personality Disorder & 103 & Mild Cognitive Disorder & 83 & Mild Cognitive Disorder & 52 & Alcohol Use Disorders & 17 \\
\hline Mild Cognitive Disorder & 97 & Personality disorders & 79 & Personality disorders & 43 & Personality disorders & 13 \\
\hline Postpartum depression & 74 & Postpartum depression & 59 & Postpartum Depression & 34 & Postpartum Depression & 13 \\
\hline Panic disorder & 68 & Panic disorder & 40 & Tobacco Use Disorders & 16 & Tobacco Use Disorders & 5 \\
\hline Phobias & 56 & Phobias & 35 & Phobias & 13 & Sleep disorders & 5 \\
\hline Schizoaffective Disorder & 51 & Schizoaffective Disorder & 32 & Panic disorder & 13 & Premature ejaculation & 5 \\
\hline Sexual dysfunction & 41 & Tobacco Use Disorders & 30 & Schizoaffective Disorder & 11 & Phobias & 4 \\
\hline Tobacco Use Disorders & 37 & Sexual dysfunction & 23 & Sexual dysfunction & 11 & Sexual dysfunction & 4 \\
\hline
\end{tabular}

Figure 3. Distribution of randomized-controlled trials published in Pubmed per top 20 ICD-10 mental and behavioral disorders, within different time frames

showing a decrease rate in 2017-2018. Recently, there has been some discussion of the possible alternatives to RCTs because of their high costs, long duration, and limited generalizability (Franklin et al., 2017). Real-world studies (longitudinal healthcare databases and registries) could be possible substitutes for RCTs in some situations (Franklin et al., 2017). In addition, there are also changing and stricter regulatory requirements that may make RCTs more problematic (e.g., tighter rules for placebo controlled RCTs; careful risk assessment), compared to observational and naturalistic studies (McNamara et al., 2019). In addition, there are fewer new drugs coming in the market since the beginning of the last decade that require their study using RCTs (Vogel, 2002).

Pharmaceutical companies have been investing less in research and development of new medications, partially because of the pharmaceutical price controls allowed by some countries to avoid the constraints of patent agreements (Vogel, 2002). The emerging new diagnoses listed in DSM-5 and ICD-11 could also play a role in attracting RCTs more than the ICD-10 and DSM-IV.

The extent of affective disorders' research probably reflects the extent of diagnosis of depression within the context of common mental disorders (Bhana et al., 2019), increased mental health literacy and recognition of depressive symptoms by the general population (Lauber et al., 2005), indications for various agents extending into this diagnostic area (Machmutow et al.,
2019), as well as the emergence of novel electrical stimulation techniques/technology and novel agents, such as ketamine (Huang et al., 2017). Delirium and mild cognitive disorder have moved up in the rankings over time, which may have to do with an increasing awareness of the need to clearly delineate medical causes rather than psychiatric only (Estrada-Orozco et al., 2018; Fowler, 2019), with consequent impact on use of resources and patient care (Fowler, 2019), and an increasing emphasis on early intervention for mild cognitive disorder as a means of preventing progression to dementia (Estrada-Orozco et al., 2018).

The proportional decrease with regard to psychotic disorders may be paralleled by the increase in organic disorders' research, boosted by dementia, delirium and mild cognitive disorder, notwithstanding that schizophrenia remains the $2^{\text {nd }}$ most investigated disorder, having several new medications on the market (Citrome, 2016). The decline of alcohol use disorders' investigation through RCTs, when it remains a public health issue, may be due to the lack of new medication candidates for clinical trials (Canella et al., 2019). A considerable amount of research with various molecules were tested for alcohol use disorders, which, in most cases, did not reach significant clinical efficacy to justify further studies, despite promising preclinical data (Canella et al., 2019).

Personality disorders are difficult to study owing to the diagnostic overlap in terms of clinical features (Wang et al., 
2019), frequent comorbidity, difficulty in recruitment and retention in treatment (Gamache et al., 2018), and also the lack of pharmacological efficacy in treatment (Bellino et al., 2011). The area of personality pathology is one of the least studied with RCT methodology, despite the evidence that such pathology accounts for around $10 \%$ of the population. The RCT's that have been performed are almost exclusively in borderline personality disorder, a condition that is an outlier in the group and might well be classified elsewhere (Tyrer, 2009; Newton-Howes et al., 2014). This is partly due to the uncertainty around effective interventions but is also influenced by lack of pharmaceutical interest. As personality pathology is associated with a poor response to treatment for common conditions such as anxiety and depression (Skodol et al., 2014), it should be addressed as an important aspect of comorbidity in randomised studies. Similar issues could be even more important for mental retardation (Cook et al., 1992; Hemayattalab et al., 2010; Artigas-Pallarés, 2006) (i.e., no definitive treatment for a non-changeable presentation), added to the fact of ethical issues with recruitment for those who cannot understand the risks involved in a given RCT (Little et al., 2002).

The 'popularity' of diagnostic categories for RCTs seems to be guided by 'clinical vogue', which could be influenced by a host of factors, such as novel treatments and ease of access to patient populations. It is not necessarily linked to the actual need as indicated by prevalence and/or burden of disorders and public health impact (e.g., alcohol use disorders). Based on the WHO treatment guideline for the most important ICD-10 mental and behavioural disorders (WHO, 1996), the following would deserve more attention from further RCTs: acute/ transient psychosis; mixed anxiety and depression; adjustment disorder; dissociative and conversion disorders; somatization; hypochondria; and neurasthenia. Dissociative disorders, including dissociative identity disorder, are almost completely neglected in the RCT literature (Simeon \& Knutelska, 2005; Ross, 2005). Another critical issue is connected with the pharmaceutical industry because they support many RCTs. In this context, many papers have been published, which favour pharmaceutical agents (psychotropics) that can have an impact on the clinical guidelines changing (Stuhec et al., 2015). Finally, most patients with mental health problems have been treated with more medications and have more mental and/or somatic diseases. In this point of view, RCTs are not always representative for this population, which is even more important in elderly patients. More real clinical patients are often covered with observational clinical trials (Castaldelli-Maia et al., 2018b; Holzapfel \& Szabo, 2018), which are very important especially in elderly patients with mental disorders (Stuhec \& Gorenc, 2019).
There are also several barriers conduct and publish psychiatric research, especially for younger colleagues (Koelkebeck et al., 2019).

\section{Limitations}

The present study used only the Pubmed database. Whilst not necessarily comprehensive, as there are other important databases (Web of Science, GoogleScholar, Scopus), it likely provides a good reflection of the status quo given Pubmed's standing as the most important medical database as well as the number of articles indexed. In addition, using data sources beyond PubMed would probably only modestly impact the results (Halladay et al., 2015). Despite not including Englishlanguage studies only, Pubmed may exclude many publications of local relevance published locally, without an assumption of a lower scientific standard, which may be a mistake (i.e., potential scientific hegemony of English-language studies). Moreover, it is important to note that ICD-10 is indeed a universal system. The exclusion of studies that did not mention randomized or randomised within the title could have also biased our results. However, it is very rare to find a study like this in Pubmed database. In addition, this selection bias may have affected all the groups of mental disorders in a similar rate. Unfortunately, the present study did not assess whether the pharmaceutical industry funded the included RCTs. Also, there was no RCT quality assessment. Finally, because we only looked at ICD 10 diagnoses, mental health conditions like suicide, catatonia and aggression, which are equally important psychiatric issues, were not included.

\section{CONCLUSION}

Despite showing an increase in the first 15 years of the 20year study period, the last 5 years was marked by a slowdown in psychiatric RCTs, similar to the other medical areas. Novel treatments, ease of access to patient populations, and 'clinical vogue, seem to be more important to guide the conducting of RCTs than the actual need as indicated by prevalence and/ or burden of disorders and public health impact. Some areas are privileged (affective, psychotic and organic disorders) potentially to the detriment of others (substance use, personality disorders and mental retardation). Regarding specific disorders, acute/transient psychosis; mixed anxiety and depression; adjustment disorder; dissociative and conversion disorders; somatization; hypochondria; and neurasthenia, would likely warrant future RCTs. Clinical researchers and scientific journal editors should give special attention to the less studied areas and disorders, when considering conducting and publishing RCT studies, respectively. 


\section{ACKNOWLEDGEMENTS}

None

\section{AUTHORS' CONTRIBUTION}

Authors JMC-M, MRB, DL-T, PSC, AC, PJT, RH, and CPD designed the study. JMCM and CPS wrote protocol, managed data collection, and created the databank. Authors JMC-M, MRB, DL-T, PSC, AC, PJT, RH, and CPD managed the literature searches and summaries of previous related work. JMC-M, RH, and CPS undertook the statistical analysis. Author JMC-M, MRB, DL-T, PSC, AC, PJT, RH, and CPD wrote the first draft of the manuscript. All authors contributed to and have approved the final manuscript.

\section{REFERENCES}

Allison, L., \& Moncrieff, J. (2014). 'Rapid tranquillisation': an historical perspective on its emergence in the context of the development of antipsychotic medications. History of psychiatry, 25(1), 57-69.

Artigas-Pallarés, J. (2006). Pharmacological treatment of mental retardation. Revista de Neurologia, 42(S1): S109-S115.

Bellino, S., Rinaldi, C., Bozzatello, P., \& Bogetto, F. (2011). Pharmacotherapy of borderline personality disorder: A systematic review. Current medicinal chemistry, 18(22), 3322-3329.

Bhana, A., Mntambo, N., Gigaba, S. G., Luvuno, Z. P. B., Grant, M., Ackerman, D., ... \& Petersen, I. (2019). Validation of a brief mental health screening tool for common mental disorders in primary healthcare. SAMJ: South African Medical Journal, 109(4), 278-283.

Cannella, N., Ubaldi, M., Masi, A., Bramucci, M., Roberto, M., Bifone, A., \& Ciccocioppo, R. (2019). Building better strategies to develop new medications in Alcohol Use Disorder: Learning from past success and failure to shape a brighter future. Neuroscience \& Biobehavioral Reviews [Epub ahead of print].

Castaldelli-Maia, J. M., Andrade, L. H., Keyes, K. M., Cerdá, M., Pilowsky, D. J., \& Martins, S. S. (2016). Exploring the latent trait of opioid use disorder criteria among frequent nonmedical prescription opioid users. Journal of psychiatric research, 80 , 79-86.

Castaldelli-Maia, J. M., Andrade, L. H., Storr, C. L., Viana, M. C., Andrade, A. G., \& Martins, S. S. (2018a). The latent trait of ICD-

\section{ETHICAL APPROVAL}

Ethical Approval not needed - Bibliometric Study

\section{FUNDING}

None

\section{INFORMED CONSENT}

Informed Consent not needed - Bibliometric Study

\section{STUDY REGISTRATION}

Study Registration not needed - Bibliometric Study
11 nicotine dependence criteria: Dimensional and categorical phenotypes. Psychiatry research, 266, 275-283.

Castaldelli-Maia, J. M., Martins, S. S., \& Walker, N. (2018b). The effectiveness of Cytisine versus Nicotine Replacement Treatment for smoking cessation in the Russian Federation. The International journal on drug policy, 58, 121-125.

Castaldelli-Maia, J. M., Wang, Y. P., Borges, G., Silveira, C. M., Siu, E. R., Viana, M. C., ... \& Andrade, L. H. (2015). Investigating dimensionality and measurement bias of DSM-5 alcohol use disorder in a representative sample of the largest metropolitan area in South America. Drug and alcohol dependence, 152, 123130.

Citrome, L. (2016). Emerging pharmacological therapies in schizophrenia: what's new, what's different, what's next?. CNS spectrums, 21(S1), 1-12.

Cook Jr, E. H., Rowlett, R., Jaselskis, C., \& Leventhal, B. L. (1992). Fluoxetine treatment of children and adults with autistic disorder and mental retardation. Journal of the American Academy of Child \& Adolescent Psychiatry, 31(4), 739-745.

Corrigan, P. W., Watson, A. C., Heyrman, M. L., Warpinski, A., Gracia, G., Slopen, N., \& Hall, L. L. (2005). Structural stigma in state legislation. Psychiatric Services, 56(5), 557-563.

Degenhardt, L., Bharat, C., Bruno, R., Glantz, M. D., Sampson, N. A., Lago, L., ... \& Caldas-de-Almeida, J. M. (2019). Concordance between the diagnostic guidelines for alcohol and cannabis 
use disorders in the draft ICD-11 and other classification systems: analysis of data from the WHO's World Mental Health Surveys. Addiction, 114(3), 534-552.

DeLuca, J. S., \& Clement, T. (2017). Does individual stigma predict mental health funding attitudes? Toward an understanding of resource allocation and social climate. The Israel journal of psychiatry and related sciences, 54(1), 6-17.

Estrada-Orozco, K., Bonilla-Vargas, K., Cruz, F., Mancera, O., Ruiz, M., Alvarez, L., ... \& Arboleda, H. (2018). Cognitive assessment test: validation of a short cognitive test for the detection of mild cognitive disorder. International Journal of Alzheimer's Disease, 2018, 3280621.

Falagas, M. E., Pitsouni, E. I., Malietzis, G. A., \& Pappas, G. (2008). Comparison of PubMed, Scopus, web of science, and Google scholar: strengths and weaknesses. The FASEB journal, 22(2), 338-342.

Fowler, B. M. (2019). Clinical Education to Decrease Perceived Barriers to Delirium Screening in Adult Intensive Care Units. Critical care nursing quarterly, 42(1), 41-43.

Frandsen, T. F., Eriksen, M. B., Hammer, D. M. G., \& Christensen, J. B. (2019). PubMed coverage varied across specialties and over time: a large-scale study of included studies in Cochrane reviews. Journal of clinical epidemiology, 112, 59-66.

Franklin, J. M., \& Schneeweiss, S. (2017). When and how can real world data analyses substitute for randomized controlled trials?. Clinical Pharmacology \& Therapeutics, 102(6), 924-933.

Gamache, D., Savard, C., Lemelin, S., Côté, A., \& Villeneuve, É. (2018). Premature Termination of Psychotherapy in Patients With Borderline Personality Disorder: A Cluster-Analytic Study. The Journal of nervous and mental disease, 206(4), 231-238.

Grossman, J., \& Mackenzie, F. J. (2005). The randomized controlled trial: gold standard, or merely standard?. Perspectives in biology and medicine, 48(4), 516-534.

Halladay, C. W., Trikalinos, T. A., Schmid, I. T., Schmid, C. H., \& Dahabreh, I. J. (2015). Using data sources beyond PubMed has a modest impact on the results of systematic reviews of therapeutic interventions. Journal of clinical epidemiology, 68(9), 1076-1084.

Harris, M., Chandran, S., Chakraborty, N., \& Healy, D. (2005). The impact of mood stabilizers on bipolar disorder: the 1890s and 1990s compared. History of Psychiatry, 1614), 423-434.
Hasan, A., Bandelow, B., Yatham, L. N., Berk, M., Falkai, P., Möller, H. J., ... \& WFSBP Guideline Task Force Chairs. (2019). WFSBP guidelines on how to grade treatment evidence for clinical guideline development. The World Journal of Biological Psychiatry, 20(1), 2-16.

Hemayattalab, R., \& Movahedi, A. (2010). Effects of different variations of mental and physical practice on sport skill learning in adolescents with mental retardation. Research in developmental disabilities, 31(1), 81-86.

Hockenberry, J. M., Joski, P., Yarbrough, C., \& Druss, B. G. (2019). Trends in treatment and spending for patients receiving outpatient treatment of depression in the United States, 1998-2015. JAMA psychiatry, 76(8), 810-817.

Holzapfel, E. M., \& Szabo, C. P. (2018). Pharmacotherapy prescribing patterns in the treatment of bipolar disorder in a South African outpatient population. Global Psychiatry, 1(2), 39-51.

Hoogendam, A., de Vries Robbe, P. F., Stalenhoef, A. F., \& Overbeke, A. J. P. (2009). Evaluation of PubMed filters used for evidencebased searching: validation using relative recall. Journal of the Medical Library Association: JMLA, 97(3), 186.

Huang, Y. J., Lane, H. Y., \& Lin, C. H. (2017). New treatment strategies of depression: based on mechanisms related to neuroplasticity. Neural plasticity, 2017, 4605971.

Koelkebeck, K., Stefanovic, M. P., Frydecka, D., Palumbo, C., Andlauer, O., Riese, F., ... \& da Costa, M. P. (2019). Barriers and facilitators to conducting research by early career psychiatrists: a literature review. Global Psychiatry, 2(2), 135-153.

Koskinen, J., Isohanni, M., Paajala, H., Jääskeläinen, E., Nieminen, P., Koponen, H., ... \& Miettunen, J. (2008). How to use bibliometric methods in evaluation of scientific research? An example from Finnish schizophrenia research. Nordic journal of psychiatry, 62(2), 136-143.

Kupfersmid, J. (2019). Freud's Clinical Theories Then and Now. Psychodynamic psychiatry, 47(1), 81-97.

Lauber, C., Ajdacic-Gross, V., Fritschi, N., Stulz, N., \& Rössler, W. (2005). Mental health literacy in an educational elite - an online survey among university students. BMC Public Health, 5(1), 44.

Little, J. D., McFarlane, J., \& Ducharme, H. M. (2002). ECT use delayed in the presence of comorbid mental retardation: a review of clinical and ethical issues. The journal of ECT, 18(4), 218-222. 
Liu, B., Liu, J., Wang, M., Zhang, Y., \& Li, L. (2017). From serotonin to neuroplasticity: evolvement of theories for major depressive disorder. Frontiers in cellular neuroscience, 11, 305.

López-Muñoz, F., Tracy, D. K., Povedano-Montero, F. J., Breedvelt, J., García-Pacios, J., Fernández-Martín, M. P., ... \& Álamo, C. (2019). Trends in the scientific literature on atypical antipsychotic drugs in the United Kingdom: a bibliometric study. Therapeutic advances in psychopharmacology, 9, 2045125318820207.

Machmutow, K., Meister, R., Jansen, A., Kriston, L., Watzke, B., Härter, M. C., \& Liebherz, S. (2019). Comparative effectiveness of continuation and maintenance treatments for persistent depressive disorder in adults. Cochrane Database of Systematic Reviews, (5), CD012855.

Manca, A., Cugusi, L., Dvir, Z., \& Deriu, F. (2017). PubMed should raise the bar for journal inclusion. The Lancet, 390(10096), 734735.

McNamara, C., Engelhardt, N., Potter, W., Yavorsky, C., Masotti, M., \& Di Clemente, G. (2019). Risk-Based Data Monitoring: Quality Control in Central Nervous System (CNS) Clinical Trials. Therapeutic innovation \& regulatory science, 53(2), 176182.

Möller-Leimkühler, A. M., Möller, H. J., Maier, W., Gaebel, W., \& Falkai, P. (2016). EPA guidance on improving the image of psychiatry. European archives of psychiatry and clinical neuroscience, 266(2), 139-154.

Newton-Howes, G., Tyrer, P., Johnson, T., Mulder, R., Kool, S., Dekker, J., \& Schoevers, R. (2014). Influence of personality on the outcome of treatment in depression: systematic review and metaanalysis. Journal of Personality Disorders, 28(4), 577-593.

Overholser, J. C. (2002). Treatments for depression: wisdom imparted from treatments discarded. The International Journal of Psychiatry in Medicine, 32(4), 317-336.

Reed, G. M. (2010). Toward ICD-11: Improving the clinical utility of WHO's International Classification of mental disorders. Professional Psychology: Research and Practice, 41(6), 457.

Reed, G. M. (2018). Progress in developing a classification of personality disorders for ICD-11. World Psychiatry, 17(2), 227-229.

Ross, C. A. (2005). A proposed trial of dialectical behavior therapy and trauma model therapy. Psychological reports, 96(S3), 901-911.
Sedler, M. J. (2016). Medicalization in psychiatry: the medical model, descriptive diagnosis, and lost knowledge. Medicine, Health Care and Philosophy, 19(2), 247-252.

Shankman, S. A., Funkhouser, C. J., Klein, D. N., Davila, J., Lerner, D., \& Hee, D. (2018). Reliability and validity of severity dimensions of psychopathology assessed using the Structured Clinical Interview for DSML5 (SCID). International journal of methods in psychiatric research, 27(1), e1590.

Simeon, D., \& Knutelska, M. (2005). An open trial of naltrexone in the treatment of depersonalization disorder. Journal of clinical Psychopharmacology, 25(3), 267-270.

Skodol, A. E., Geier, T., Grant, B. F., \& Hasin, D. S. (2014). Personality disorders and the persistence of anxiety disorders in a nationally representative sample. Depression and Anxiety, 31(9), 721-728.

Stuhec, M., \& Gorenc, K. (2019). Positive impact of clinical pharmacist interventions on antipsychotic use in patients on excessive polypharmacy evidenced in a retrospective cohort study. Global Psychiatry, 2(2), 155-163.

Stuhec, M., Munda, B., Svab, V., \& Locatelli, I. (2015). Comparative efficacy and acceptability of atomoxetine, lisdexamfetamine, bupropion and methylphenidate in treatment of attention deficit hyperactivity disorder in children and adolescents: a meta-analysis with focus on bupropion. Journal of affective disorders, 178, 149159.

Thoma, N., Pilecki, B., \& McKay, D. (2015). Contemporary cognitive behavior therapy: A review of theory, history, and evidence. Psychodynamic Psychiatry, 43(3), 423-461.

Tyrer, P. (2009). Why borderline personality disorder is neither borderline nor a personality disorder. Personality and Mental Health, 3(2), 86-95.

Vogel, R. J. (2002). Pharmaceutical patents and price controls. Clinical therapeutics, 24(7), 1204-1222.

Wang, J., Chen, Z., Sun, W., Zheng, Y., Hui, L., Liu, X., ... \& Zhang, T. (2019). A Strategy to Address High Comorbidity of Personality Disorders in a Chinese Population: A Principal and Subordinate Diagnostic Model. Psychiatry, 82(3), 272-282.

World Health Organization. (1992). The ICD-10 Classification of Mental and Behavioural Disorders: Clinical Descriptions and Diagnostic Guidelines (Vol. 1). World Health Organization. 
World Health Organization. (1993). The ICD-10 classification of mental and behavioural disorders: diagnostic criteria for research (Vol. 2). World Health Organization.

World Health Organization. (1996). Diagnostic and management guidelines for mental disorders in primary care: ICD-10. Chapter 5, Primary care version. World Health Organization. 